\section{T-cell large granular lymphocyte leukemia transfomation into aggressive T-cell Iymphoma: a report of two cases with molecular characterization}

T-cell large granular lymphocyte (LGL) leukemia, a clonal expansion of cytotoxic $\mathrm{CD}^{+} \mathrm{T}$ cells, represents $5 \%$ of lymphoproliferative disorders. Clinical features include neutropenia causing recurrent bacterial infections, anemia of diverse mechanisms, and autoimmune diseases such as rheumatoid arthritis. T-LGL leukemia is an indolent disease, and related deaths are mainly due to infections. ${ }^{1}$ We describe two cases of clonal evolution of
T-LGL leukemia into aggressive T-cell lymphoma.

In 2010, a 55-year-old Caucasian woman presented with isolated lymphocytosis at $8 \times 10^{9} / 1$. Physical examination was normal. Circulating cells were typical LGLs with a $\mathrm{CD}^{+}, \mathrm{CD}^{+}, \mathrm{CD}^{+}, \mathrm{CD}^{+}, \mathrm{CD}^{-}, \mathrm{CD}^{-} 6^{-}, \mathrm{CD}^{-} 6^{-}, \mathrm{CD}^{-} 7^{-}$ phenotype with a clonal TCR- $\gamma$ gene rearrangement. Two years later, she presented with fatigue, night sweats, hepatosplenomegaly and enlarged cervical and axillary lymph nodes. The blood cell count showed: leucocytes $132 \times 10^{\%} / 1$, neutrophils $17 \times 10^{9} / 1$, platelets $314 \times 10^{9} / 1$, hemoglobin $13.3 \mathrm{~g} / \mathrm{dl}$. Tumor lysis syndrome and lactic acidosis were detected. Blood smear revealed a lymphocyte population made of large cells with abundant, mod-
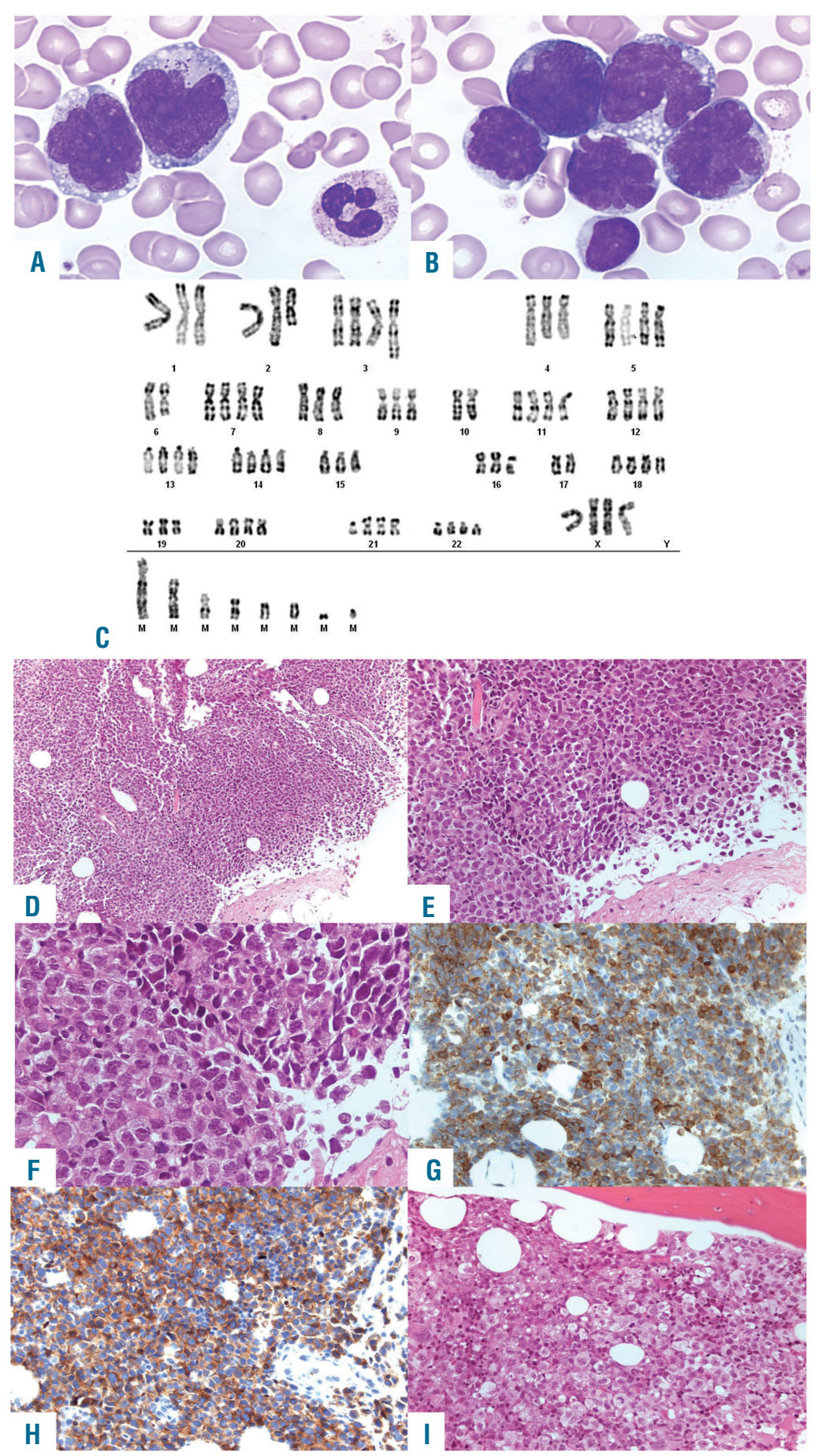

Figure 1. Cytologic, cytogenetic and pathological analysis of patient 1. (A-B) Blood smear revealing a lymphocyte population made of large cells with abundant, moderately basophilic cytoplasm containing granules, vacuoles and irregular nuclei (MGG, 100x). (C) Complex "near-tetraploid" karyotype (peripheral blood): $79 \sim 89, X X X X,-1,-2, \operatorname{add}(2)(q 23)$,3,der(3)t(1;3)(q31; q27),-4,-6,-6,del(6)(q?q?),$8,-9,-10,-10,-15,-16,-17,-17,-19$, i(21)(q10)x3,+7 14mar[cp16]. Ish 17p13.1 (TP53,17qtel)x2, mar(TP53-,17qtel++)x3[8]. (D-E-F) Lymph node biopsy showing massive infiltration by medium- to large-sized cells with irregular pleomorphic nuclei, and abundant cytoplasm (H\&E-safran 10x, 20x 40x). (G-H) By immunohistochemistry in the lymph node, tumor cells are $\mathrm{CD}^{+}$and $\mathrm{CD}^{+}(\mathrm{SABC}, 20 \mathrm{x})$. I: The same tumor cell population was also found in the bone marrow (H\&E-safran, 20x). 


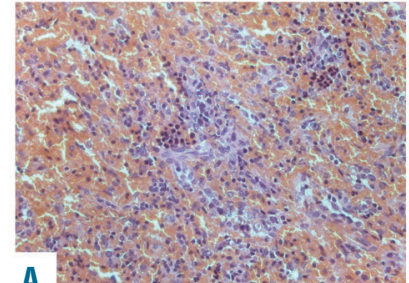

A
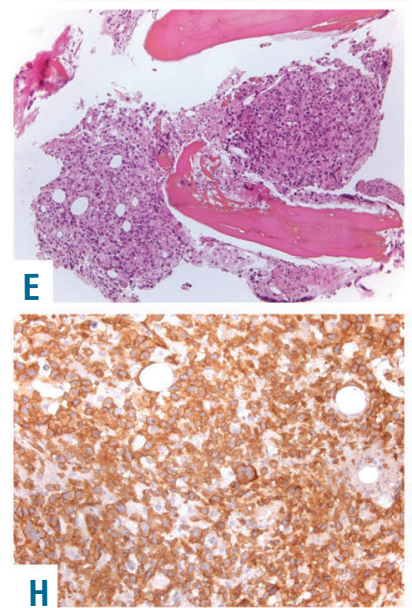
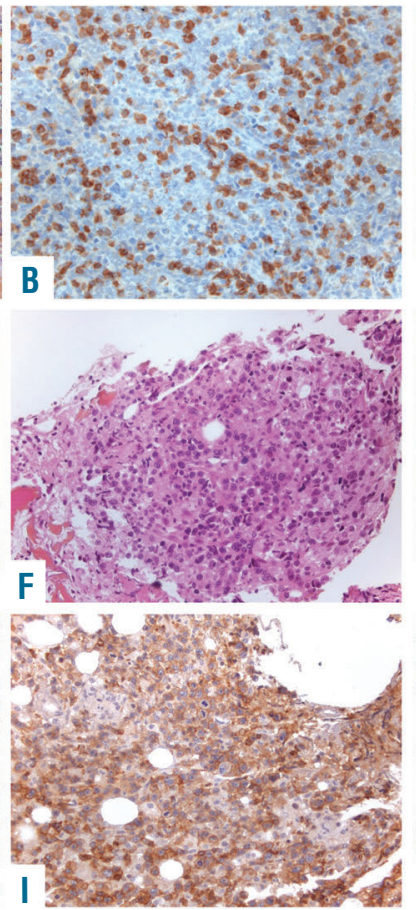
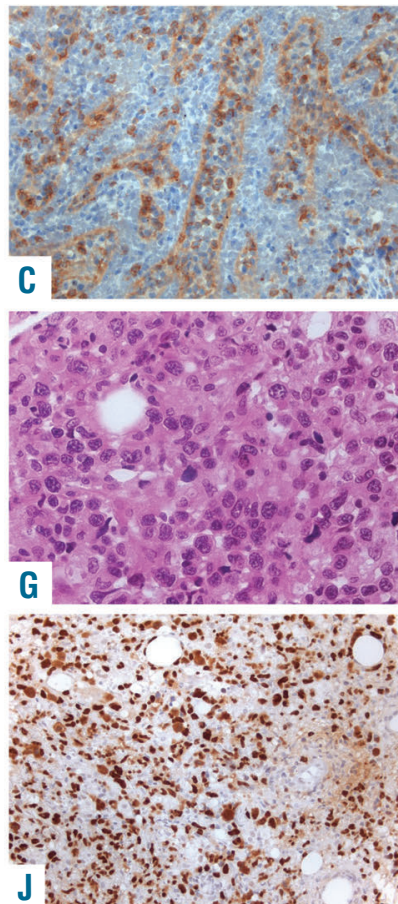

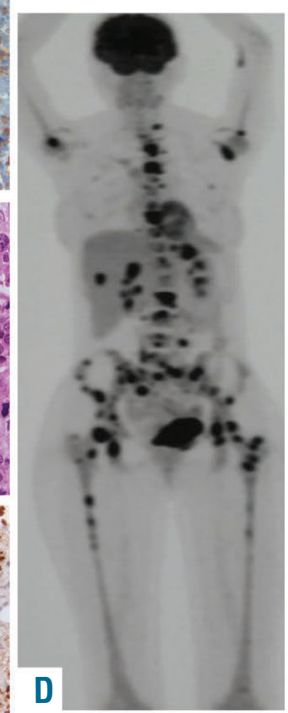

Figure 2. Cytologic, cytogenetic and pathological analysis of patient 2. (A) Splenectomy showed preserved architecture, with small- to medium-sized atypical lymphocytes infiltrating both cords and sinusoids (H\&E-safran, 20x). (B) CD3 immunostaining highlights cells within splenic sinusoids (SABC, 20x). (C) Tumor cells in the spleen, as well as approximately 60\% of T-LGL, are CD8-positive (SABC, 20x). (D) Multifocal hypermetabolic bone lesions on F-18 FDG PET/CT. (E-FG) Spinal bone (L3) biopsy showing extensive infiltration by large cells with irregular nuclei, abundant, slightly basophilic cytoplasm, along with large anaplastic cells (H\&E-safran 10x, 20x, 40x). (H-I-J) By immunohistochemistry, tumor cells in bone biopsy are $\mathrm{CD}^{+}(\mathrm{G})$ and $\mathrm{CD} 8^{+}(\mathrm{H})$ with a diffuse and intense nuclear expression of p53 (I) (SABC, 20x).

erately basophilic cytoplasm containing granules and vacuoles, and irregular nuclei (Figures $1 \mathrm{~A}-\mathrm{B}$ ). Their phenotype was identical to T-cell LGL leukemia except for a weak CD3 expression. Blood karyotype at evolution was hyperploid and complex, and FISH analysis detected 17p deletion (Figure 1C). Lymph node biopsy showed massive and diffuse infiltration by medium to large-sized atypical cells with irregular and pleomorphic nuclei without nucleoli and abundant cytoplasm (Figures 1D-E-F). Tumor cells were $\mathrm{CD}^{+}, \mathrm{CD}^{+}, \mathrm{CD}^{+/}, \mathrm{CD}^{+} 0^{+}$, granzyme $\mathrm{B}^{+}$and $\mathrm{TiA1}^{+}$(Figures $\left.1 \mathrm{G}-\mathrm{H}\right)$. An identical proliferation was found in the bone marrow (Figure 1I). The same clonal TCR rearrangement was detected, confirming that $\mathrm{CD}^{+} \mathrm{T}$-LGL leukemia had transformed into aggressive T-cell lymphoma. Two mutations were found in the TP53 gene: the first (R282W in exon 8) was detected in a blood sample from 2010 with a variant allelic frequency (VAF) of $6 \%$ and was deleterious according to IARC TP53 database. ${ }^{2}$ At evolution, R282W was only $1 \%$ whereas the second mutation (V157I in exon 5) was detected in blood samples in 2010 and at transformation, but its germline origin was confirmed by a skin biopsy. No mutation of STAT3 or STAT $5 B$ genes was detected.

The patient received 2 cycles of $\mathrm{CHOP}, 3$ cycles of CHOEP and one cycle of DHAX without success. She then achieved complete remission after one cycle of highdose Methotrexate and L-Asparaginase which made it possible to perform an autologous stem cell transplant (ASCT) with BEAM conditioning, but disease progressed rapidly after ASCT and the patient died.

A 30-year-old Caucasian woman presented with isolated neutropenia in 2011 (neutrophils $0.76 \times 10^{9} / 1$ and lym- phocytes $\left.2.8 \times 10^{9} / 1\right)$. LGL were detected on blood smear, representing $61 \%\left(1.7 \times 10^{\circ} / 1\right)$ of total lymphocytes, with a $\mathrm{CD}^{+}, \mathrm{CD}^{+}, \mathrm{CD}^{-}$, weak CD5, partial CD56 and CD57 phenotype and clonal TCR- $\gamma$ gene rearrangement which confirmed the diagnosis of CD8 ${ }^{+} \mathrm{T}-\mathrm{LGL}$ leukemia. There was no TP53 gene mutation, but a heterozygous STAT3 mutation (Y640F in exon 21) was detected. Physical examination and blood biochemistry were normal.

In 2013, she presented with fatigue, fever, hepatosplenomegaly and pericarditis. Blood cell count showed: leucocytes $1.9 \times 10^{9} / 1$, neutrophils $0.34 \times 10^{9} / 1$, lymphocytes $0.78 \times 10^{9} / 1$, platelets $426 \times 10^{9} / 1$, hemoglobin $6.4 \mathrm{~g} / \mathrm{dl}$. Intramedullary hemolytic anemia related to hemophagocytosis and disseminated intravascular coagulation (DIC) were present. A liver biopsy revealed lobular hepatitis without lymphoid infiltration. No viral infection was found to explain hemophagocytosis. She received two cycles of etoposide with corticosteroids and improved quickly but relapsed within a month. She then received etoposide and cyclophosphamide with intravenous gamma-globulin, allowing her condition to improve for a month before relapsing. Splenectomy, liver and bone marrow biopsies were performed. Splenic infiltrates accumulating in the sinusoids and cords with medium-sized T-lymphocytes infiltrating the red pulp were detected with a $\mathrm{CD}^{+}, \mathrm{CD}^{+}, \mathrm{CD}^{+}, \mathrm{CD}^{-}, \mathrm{TIA}_{-1}^{+}, \mathrm{CD}^{+} 7^{+}$ phenotype (Figures 2A-B-C). Bone marrow biopsy showed weak interstitial and intra-vascular infiltration by the same clonal LGL population. This third episode was treated with etoposide, cyclophosphamide and corticosteroids, but the patient relapsed three months later. F-18 FDG PET/CT imaging showed hypermetabolic lesions on 
L3 vertebra, left rib, sternum and iliac crest (Figure 2D). L3 vertebra biopsy showed extensive infiltration by large cells with irregular nuclei, abundant slightly basophilic cytoplasm and large anaplastic cells (Figures 2E-F-G). Their phenotype was $\mathrm{CD}^{+}, \mathrm{CD}^{+}, \mathrm{CD}^{+}, \mathrm{CD} 30^{+}, \mathrm{TiA}-1^{+}$, Granzyme B ${ }^{+}, \mathrm{P}^{2} 3^{+}, \mathrm{CD} 2^{+/}, \mathrm{CD}^{+} 6^{+}, \mathrm{CD}^{+} 7^{+}, \mathrm{CD}^{-}, \mathrm{CD} 20^{-}$, CD79a', ALK ${ }^{-}$(Figure 2H-I-G). The same clonal TCR rearrangement was confirmed. TP53 gene analysis detected a deleterious P151T mutation in exon 5 (VAF $85 \%$ ) which was not detected at diagnosis. STAT3 gene analysis revealed that the heterozygous mutation detected in 2011 had turned into a homozygous one at transformation. An additional mutation in BCOR was found (p.G599fs, VAF 57\%).

The patient received one cure of Brentuximab-Vedotin (BV)-CHEP and one cure of BV-CHOEP, but disease progressed. Treatment was changed to BV-DHAX with etoposide and infliximab because of recurrent episodes of DIC and hemophagocytosis, but the patient died of disease progression.

T-cell LGL leukemia is an indolent disease with survival rates around $70 \%$ at 10 years. ${ }^{1}$ Very rare cases of aggressive forms of T-cell LGL leukemia have been reported. ${ }^{3,4}$

Whilst the median age at diagnosis of T-LGL leukemia is $60,{ }^{1}$ our patients were 55 and 30 years old, respectively. In the transformed cases published, age at diagnosis of aggressive forms is around 41 years, ${ }^{5}$ which is concordant with our experience. The sex ratio, which is 1:1 in T-cell LGL leukemia, may be 2:1 in favor of women in aggressive forms, and our patients were female.

In our cases, demonstration of clonal evolution of TLGL leukemia in aggressive lymphoma came from the phenotypic conservation of the cell of origin: $\mathrm{CD}^{+}, \mathrm{CD}^{+}$ in patient 1 , which is an uncommon phenotype, and $\mathrm{CD}^{+}$in patient 2 expressing the cytotoxic markers granzyme B and TiA1, but essentially from a common clonal TCR-gamma rearrangement. Previously reported cases generally describe: appearance of acute B signs, rapidly enlarging lymph-nodes, infiltration of amygdales and subcutaneous adipose tissues, cytopenia due to bone marrow infiltration, hepatosplenomegaly in which the architecture of the spleen and the liver with persistent infiltration by small T cells of the LGL clone is usually conserved.

In most reported cases, the morphologic aspect does not differ from that observed in peripheral T-cell lymphomas. ${ }^{6}$ In NOS T-cell lymphoma, ${ }^{7}$ malignant cells are large pleomorphic cells with irregular nuclei, immature chromatin and often a well-defined nucleolus associated with abundant and clear cytoplasm with detection of cells in mitosis. Similar morphologic aspects were present in our cases.

Concerning the biological characteristics associated with transformation, only cytogenetic analysis was previously reported in one case with complex cytogenetics. ${ }^{8}$ In patient 1 , complex karyotype, near tetraploid, with del $17 p$ deletion was detected. No molecular study has previously been reported. It is suggested that T-cell LGL leukemia arises from antigenic stimulation of effector memory cytotoxic T-cells leading to up-regulation of the JAK-STAT3 signaling pathway that drives sub-clone selection with acquired STAT3 mutations, which are reported in $28-75 \%$ of LGL leukemia. ${ }^{1}$ In $2 \%$ of cases, STAT $5 b$ mutations are found. ${ }^{9}$ In patient 1 , no mutation in the STAT3 or STAT5b genes was detected, whereas patient 2 had a heterozygous STAT3 mutation which turned into a homozygous mutation at transformation. The biological consequence of this evolution is unknown. We also detected acquired mutations in the TP53 gene that caused protein inactivation: $\mathrm{R} 282 \mathrm{~W}^{10}$ in exon 8 since diagnosis in patient 1 , and P151T in exon 5 in patient 2 only at transformation. In patient 1 , complete loss of the p53 pathway was present at the stage of aggressive T-cell lymphoma. The role of the mutations in the process of transformation is unknown, but they probably participated in chemo-resistance and chromosomal instability. For patient 2, a BCOR mutation was present at transformation. It is known that $50 \%$ of mice missing BCOR (exon 4 deletion) develop T-cell acute lymphoblastic leukemia (T-ALL) providing evidence of a tumor suppressor role for BCOR in T-cell malignancies, and cooperation between BCOR mutation and inactive TP53 allele in the development of T-ALL was documented. ${ }^{11}$ Furthermore, in a cohort of extra-nodal NK/T-cell lymphoma, nasal type, $B C O R$ was the second most frequent mutated gene after STAT3, suggesting a role of BCOR mutation in lymphoma pathogenesis together with alteration of the JAK/STAT cascade. ${ }^{12}$ The T-cell lymphoma was refractory to almost all chemotherapy regimens in our 2 patients. In patient 1 , complete remission was only obtained after one course of Methotrexate and LAsparaginase, suggesting that tumor survival was dependent on asparagine and/or glutamine metabolism. In patient 2, all treatments were ineffective, including combination of chemotherapy to BV currently used in peripheral $\mathrm{CD}^{+} 0^{+} \mathrm{T}$-cell lymphomas with encouraging results. ${ }^{13}$ From these 2 cases and the few already published, we cannot propose a strategy of first-line treatment. Even high dose chemotherapy and auto-grafting in patient 1 was unsuccessful. Alternatively, chemotherapy induction regimens used in T-cell ALL were recommended to propose allografting in younger patients. ${ }^{14}$ Modern immunotherapy with CAR-T cells may be more efficient, but the antigens to target need to be identified. Alternatively, the rationale for testing compounds such as APR-246, which can restore function and transcriptional activity of p53 mutated proteins, may be high. ${ }^{15}$

Maya Belhadj, 1,2 Dalila Mansour,, Sophie Kaltenbach, ${ }^{3,4}$ Benedicte Deau-Fischer, ${ }^{2,3}$ Patricia Franchi, ,,3 Jérôme Tamburini, 2,3,5 Nicolas Chapuis, ,3,6 Diane Damotte, ${ }^{3,7}$ Olivier Kosmider, ${ }^{3,5,6}$ Barbara Burroni, ${ }^{3,7}$ and Didier Bouscary ${ }^{2,3,5}$

${ }^{1}$ Université Paris Est-Créteil, UFR de Médecine; ${ }^{2}$ Service d'hématologie clinique, Hôpital Cochin, HUPC, Assistance Publique-Hôpitaux de Paris; ${ }^{3}$ Université Paris Descartes, Faculté de Médecine Sorbonne Paris Cité; ${ }^{4}$ Service de cytogénétique, Hôpital Necker, Assistance Publique-Hôpitaux de Paris; 'INSERM U1016, Institut Cochin, Paris; 'Service d'hématologie biologique, Hôpital Cochin, HUPC, Assistance Publique-Hôpitaux de Paris and 'Service d'anatomopathologie, Hôpital Cochin, HUPC, Assistance Publique-Hôpitaux de Paris, France

$B B$ and $D B$ contributed equally to this work.

Correspondence: MAYA BELHADJ - maya.belhadj@hotmail.fr doi:10.3324/haematol.2018.205542

Information on authorship, contributions, and financial \& other disclosures was provided by the authors and is available with the online version of this article at www. haematologica.org.

\section{References}

1. Lamy T, Moignet A, Loughran TP. LGL leukemia: from pathogenesis to treatment. Blood. 2017;129(9):1082-1094.

2. Olivier M, Eeles R, Hollstein M, Khan MA, Harris CC, Hainaut P. The IARC TP53 database: new online mutation analysis and recommendations to users. Hum Mutat. 2002;19(6):607-614.

3. Passetto Falcão R, Pinto Simões B, Garcia AB, Fonseca BA, Terra Filho J. Aggressive variant of morphologically typical T large granular lymphocyte leukemia/lymphoma lacking NK cell markers. Acta Haematol. 2000;104(2-3):110-114. 


\section{Case Reports}

4. Tagawa S, Mizuki M, Onoi U, et al. Transformation of large granular lymphocytic leukemia during the course of a reactivated human herpesvirus-6 infection. Leukemia. 1992;6(5):465-469.

5. Steinway SN, Leblanc F, Loughran TP. The pathogenesis and treatment of large granular lymphocyte leukemia. Blood Rev. 2014;28(3):87-94

6. Matutes E, Wotherspoon AC, Parker NE, Osuji N, Isaacson PG, Catovsky D. Transformation of T-cell large granular lymphocyte leukaemia into a high-grade large T-cell lymphoma. Br J Haematol. 2001;115(4):801-806

7. Swerdlow SH, Campo E, Pileri SA, et al. The 2016 revision of the World Health Organization classification of lymphoid neoplasms. Blood. 2016:127(20):2375-2390.

8. Zhang L, Ramchandren R, Papenhausen P, Loughran TP, Sokol L. Transformed aggressive $\gamma \delta$-variant T-cell large granular lymphocytic leukemia with acquired copy neutral loss of heterozygosity at 17q11.2q25.3 and additional aberrations. Eur J Haematol. 2014;93(3):260-264.

9. Rajala HLM, Eldfors S, Kuusanmäki H, et al. Discovery of somatic STAT5b mutations in large granular lymphocytic leukemia. Blood. 2013;121(22):4541-4550.
10. Calhoun S, Daggett V. Structural effects of the L145Q, V157F, and R282W cancer-associated mutations in the p53 DNA-binding core domain. Biochemistry. 2011;50(23):5345-5353.

11. Tanaka T, Nakajima-Takagi $Y$, Aoyama $K$, et al. Internal deletion of BCOR reveals a tumor suppressor function for BCOR in T lymphocyte malignancies. J Exp Med. 2017;214(10):2901-2913.

12. Lee S, Park HY, Kang SY, et al. Genetic alterations of JAK/STAT cascade and histone modification in extranodal NK/T-cell lymphoma nasal type. Oncotarget. 2015;6(19):17764-17776.

13. Fanale MA, Horwitz SM, Forero-Torres A, et al. Five-year outcomes for frontline brentuximab vedotin with $\mathrm{CHP}$ for CD30-expressing peripheral T-cell lymphomas. Blood. 2018;131(19):2120-2124.

14. Ishida $\mathrm{F}, \mathrm{Ko} \mathrm{YH}, \mathrm{Kim} \mathrm{WS}$, et al. Aggressive natural killer cell leukemia: therapeutic potential of L-asparaginase and allogeneic hematopoietic stem cell transplantation. Cancer Sci. 2012;103 (6):1079-1083.

15. Duffy MJ, Synnott NC, Crown J. Mutant p53 as a target for cancer treatment. Eur J Cancer. 2017;83258-265. 